\title{
GENERALIZED IMPEDANCE BOUNDARY CONDITION AT HIGH FREQUENCY FOR A DOMAIN WITH THIN LAYER: THE CIRCULAR CASE
}

\author{
CLAIR POIGNARD
}

\begin{abstract}
Consider a conducting disk surrounded by a thin dielectric layer submitted to an electric field at the pulsation $\omega$. The conductivity of the layer grows like $\omega^{1-\gamma}, \gamma \in[0,1]$, when the pulsation $\omega$ tends to infinity. Using a pseudodifferential approach on the torus, we build an equivalent boundary condition with the help of an appropriate factorization of Helmholtz operator in the layer. This generalized impedance condition approximates the thin membrane in the high frequency limit for small thickness of the layer. $L^{2}$-error estimates are given and we illustrate our results with numerical simulations. This work extends, in the circular geometry, previous works of Lafitte and Lebeau [7], [8], in which $\gamma$ identically equals zero.
\end{abstract}

\section{INTRODUCTION AND MAIN RESULTS}

Approximated boundary conditions for domains with thin layer have been extensively studied for Helmholtz equation at given frequency during the last twenty years; see for instance [1], [5], [3] or [2], and [10] for highly contrasted domains. In these previous works a bounded domain $\mathcal{O}$ surrounded by a thin layer is considered. The main idea of the above papers is to build a condition on $\partial \mathcal{O}$ equivalent to the layer using an asymptotic expansion of the solution to the Helmholtz equation with respect to the thickess of the layer. This asymptotic expansion is based on a suitable change of variables in the thin layer. Helmholtz operator is then written in these local coordinates, and its dependency with respect to the thinness of the layer appears explicitely.

In the high frequency regime, the analysis performed in these previous articles might not be applied, since two small parameters appear: the thinness of the layer and the inverse of the frequency. Several authors have studied the scattered field of an incident wave at high frequencies by a convex obstacle, see for instance the works of Leontovitch [9], Lafitte and Lebeau [7], Lafitte [8]. Using a WentzelKramer-Brillouin (WKB) expansion Leontovitch found the well-known impedance boundary condition, which links the electric and magnetic fields on the boundary of the obstacle:

$$
n \times E=\sqrt{\frac{\mu}{\varepsilon}} n \times(n \times H) .
$$

1991 Mathematics Subject Classification. 34E05, 34E10, 35J05.

Key words and phrases. Asymptotics, Helmholtz Equation, Thin Layer, Generalized Impedance Boundary Conditions, Fourier Analysis.

CMAP, Ecole Polytechnique. mailto: poignard@cmapx.polytechnique.fr

Published in Applicable Analysis, Vol.86, Issue 12, 2007. 
In bidimensional domain, and using Faraday's law (rot $E=\mathrm{i} \omega \mu H$ ), the Leontovitch condition becomes:

$$
\partial_{n} E-\mathrm{i} \omega \sqrt{\varepsilon \mu} E=0 .
$$

This impedance boundary condition is asymptotically equivalent to the conducting obstacle, for $\omega$ tending to infinity. In [7] Lebeau and Lafitte generalized this condition using an appropriate microlocal analysis in which the multiplication by $\omega$ is an operator of order 1 , like the derivation. In these works, it is necessary to deal with a highly conducting thin layer in the sense that the layer conductivity grows like $\omega$, whereas usual materials have constant conductivity.

Using an appropriate pseudodifferentential calculus on the torus, we generalize the previous works to the case of a layer condutivity $\sigma \omega^{1-\gamma}$, for $\gamma$ in $[0,1]$. For $\gamma=0$, our boundary condition is a high frequency limit of the Leontovitch condition. Moreover we give error estimates, which justifies the use of our generalized impedance boundary condition. Since the microlocal analysis needs technicity especially for a layer of arbitrary shape, we consider here the circular case in order to present the main ideas of our analysis.

1.1. Studied problem and notations. We consider a heterogeneous medium $\Omega_{\delta}$ composed by a conducting inner disk $\mathcal{O}$ surrounded by thin dielectric layer $\mathcal{O}_{\delta}$ with constant thickness $\delta$. This medium is submitted on its exterior boundary to an electric field of pulsation $\omega$. We study the behavior of the electric field in this medium for $\omega$ tending to infinity and $\delta$ to zero. We propose a generalized impedance boundary condition equivalent to the thin membrane and we estimate the error of our approximation. This generalized impedance boundary condition is given in terms of pseudodifferential operators on the torus $\mathbb{T}$.

Let $\gamma \in[0,1]$. The strictly positive constants $\mu_{c}$ and $\mu_{m}$ denote the respective permeabilities of $\mathcal{O}$ and of $\mathcal{O}_{\delta}$. Let $\varepsilon_{c}, \sigma_{c}, \varepsilon_{m}, \sigma_{m}$ four dielectric constants. We denote by $\widetilde{\varepsilon}_{c}$ and $\widetilde{\varepsilon}_{m}$ the respective complex permittivities of $\mathcal{O}$ and of $\mathcal{O}_{\delta}$ :

$$
\widetilde{\varepsilon}_{c}=\varepsilon_{c}+\mathrm{i} \sigma_{c}, \quad \widetilde{\varepsilon}_{m}=\varepsilon_{m}+\mathrm{i} \frac{\sigma_{m}}{\omega \gamma} .
$$

Observe that the imaginary part of $\widetilde{\varepsilon}_{c}$ is constant, hence $\mathcal{O}$ is a conducting disk, while those of $\widetilde{\varepsilon}_{m}$ decreases as $\omega^{-\gamma}$, for $\omega$ tending to zero. We denote by $\mu$ and $\widetilde{\varepsilon}$

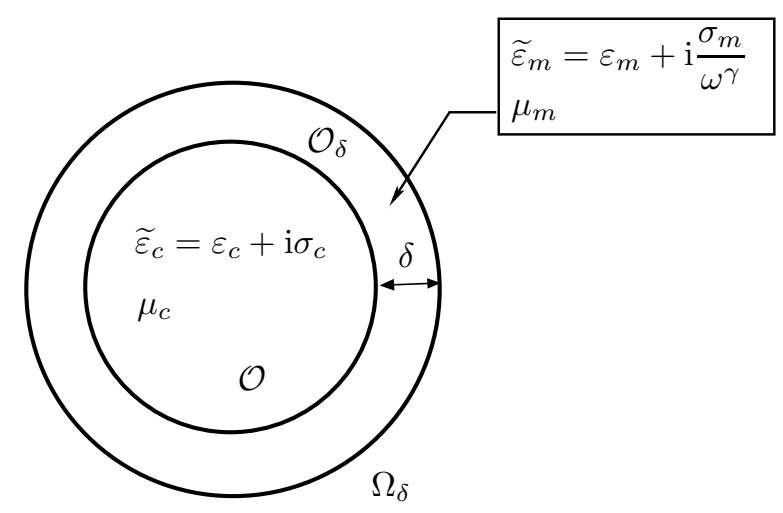

FiguRE 1. Electromagnetic parameters and geometry of the medium. 
the two following functions defined in $\Omega_{\delta}$ :

$$
\mu(x)=\left\{\begin{array}{l}
\mu_{c}, \text { in } \mathcal{O}, \\
\mu_{m}, \text { in } \mathcal{O}_{\delta},
\end{array} \quad \widetilde{\varepsilon}(x)=\left\{\begin{array}{l}
\widetilde{\varepsilon}_{c}, \text { in } \mathcal{O}, \\
\widetilde{\varepsilon}_{m}, \text { in } \mathcal{O}_{\delta} .
\end{array}\right.\right.
$$

It is also convenient to denote by $q_{m}$ and $q_{c}$ the respective products $\mu_{m} \widetilde{\varepsilon}_{m}$ and $\mu_{c} \widetilde{\varepsilon}_{c}$, and we set $\Re\left(q_{c}\right)=a_{c}, \Im\left(q_{c}\right)=b_{c}, \Re\left(q_{m}\right)=a_{m}$ and $\Im\left(q_{m}\right)=b_{m} / \omega^{\gamma}$.

Let $g$ belong to $H^{1 / 2}\left(\partial \Omega_{\delta}\right)$. Denote by $E$ the electric field, solution to Helmholtz equation in $\Omega_{\delta}$ with Dirichlet boundary condition:

$$
\begin{aligned}
& \nabla \cdot\left(\frac{1}{\mu} \nabla E\right)+\omega^{2} \widetilde{\varepsilon} E=0, \text { in } \Omega_{\delta}, \\
& \left.E\right|_{\partial \Omega_{\delta}}=g, \text { on } \partial \Omega_{\delta} .
\end{aligned}
$$

Since equality $(2 \mathrm{a})$ is written in the weak sense, the following transmission conditions hold on $\partial \mathcal{O}$ :

$$
\begin{aligned}
& \left.E\right|_{\partial \mathcal{O}} ^{+}=\left.E\right|_{\partial \mathcal{O}} ^{-}, \\
& \left.\frac{1}{\mu_{m}} \partial_{n} E\right|_{\partial \mathcal{O}} ^{+}=\left.\frac{1}{\mu_{c}} \partial_{n} E\right|_{\partial \mathcal{O}} ^{-} .
\end{aligned}
$$

Using these transmission conditions, Leontovicth impedance condition (1) becomes formally ${ }^{1}$

$$
\left.\frac{\mu_{m}}{\mu_{c}} \partial_{n} v\right|_{\partial \mathcal{O}}-\left.\mathrm{i} \omega \sqrt{q_{m}} v\right|_{\partial \mathcal{O}}=0 .
$$

Our goal is to prove and to generalize this condition for $\omega$ tending to infinity and $\delta$ to zero.

The present paper is structured as follows. In Section 2, we suitably factorize Helmholtz operator and we give precise estimates of the involved symbols. Section 3 studies the factorized problem in the thin layer. More precisely, supposing that the traces of the electric field are known on $\partial \Omega_{\delta}$ and $\partial \mathcal{O}$, we give explicitly the Fourier coefficients of the solution to the factorized problem. This leads to our generalized boundary condition. Section 4 is devoted to prove $L^{2}$-error estimates. We conclude this paper with a discussion on the impedance boundary condition for a thin layer of an arbitrary shape.

Present now our main result and our numerical simulations.

1.2. Main result. Define the following symbols:

$$
\begin{aligned}
\forall \omega & \geq 1, \eta \in[0,1], \theta \in \mathbb{T}, k \in \mathbb{Z} \\
d_{1}(\omega, \eta, k) & =\sqrt{\frac{k^{2}}{(1+\delta \eta)^{2}}-q_{m} \omega^{2}}, \quad \text { with } \Re\left(d_{1}(\omega, \eta, k)\right) \leq 0, \\
\widetilde{d}_{1} & =d_{1}-\frac{\partial_{\eta} d_{1}}{2 \delta d_{1}}, \\
d & =\widetilde{d}_{1}-\frac{1}{2(1+\delta \eta)}, \quad \widetilde{d}=\widetilde{d}_{1}+\frac{1}{2(1+\delta \eta)}
\end{aligned}
$$

\footnotetext{
${ }^{1}$ As we said above, Leontovitch condition has been shown only for $\Im\left(q_{m}\right) \geq c>0$, whereas here $\Im\left(q_{m}\right)=b_{m} \omega^{-\gamma}$ with $\gamma \in[0,1]$.
} 
Let $\mathfrak{p}$ and $\mathfrak{s}$ be defined by:

$$
\begin{aligned}
& \mathfrak{p}(\omega, k)=\left(\int_{0}^{1} e^{\delta \int_{x^{\prime}}^{1}(d+\widetilde{d})(\omega, s, k) \mathrm{d} s} \mathrm{~d} x^{\prime}\right)^{-1}, \\
& \mathfrak{s}(\omega, k)=\frac{1}{\delta} \mathfrak{p}(\omega, k) e^{\delta \int_{0}^{1} \widetilde{d}(\omega, s, k) \mathrm{d} s},
\end{aligned}
$$

and define $\mathscr{A}$ and $\mathscr{B}$ the two following pseudodifferential operators:

$$
\begin{aligned}
& \forall s \in \mathbb{R}, \forall \theta \in \mathbb{T}, \forall u \in H^{s}(\mathbb{T}), \\
& \mathscr{A} u(\theta)=\frac{1}{2 \pi} \sum_{k \in \mathbb{Z}}\left(d(\omega, 0, k)-\mathfrak{s}(\omega, k) e^{\delta \int_{0}^{1} d(\omega, s, k) \mathrm{d} s}\right) \int_{0}^{2 \pi} u(s) e^{\mathrm{i} k(\theta-s)} \mathrm{d} s, \\
& \mathscr{B} u(\theta)=\frac{1}{2 \pi} \sum_{k \in \mathbb{Z}} \mathfrak{s}(\omega, k) \int_{0}^{2 \pi} u(s) e^{\mathrm{i} k(\theta-s)} \mathrm{d} s .
\end{aligned}
$$

Denote by $\Psi$ the $\mathscr{C}^{\infty}$-diffeomorphism, which maps the torus $\mathbb{T}$ unto $\partial \mathcal{O}$. For all $s \in \mathbb{R}$, for all $v \in H^{s}(\partial \mathcal{O})$ the operators $\mathrm{A} v$ and $\mathrm{B} v$ are defined by:

$$
\begin{aligned}
& \mathrm{A} v=(\mathscr{A} v \circ \Psi) \circ \Psi^{-1}, \\
& \mathrm{~B} v=(\mathscr{B} v \circ \Psi) \circ \Psi^{-1} .
\end{aligned}
$$

Hypothesis 1.1. In this paper, we suppose:

- $\gamma \in[0,1]$.

- $\delta \rightarrow 0$.

- $\omega \rightarrow+\infty$.

- $\delta \omega^{1-\gamma / 2} \geq C_{0}$, for $C_{0}>0$ large enough, depending only on the parameters $\mu_{m}, \varepsilon_{m}$ and $\sigma_{m}$.

The hypothesis $\delta \omega^{1-\gamma / 2} \geq C_{0}$ seems to be technical and is precisely given in (37). It might be seen as an extension of the hypothesis $\delta \omega \rightarrow c_{0}>0$ of Lafitte [8]. Moreover if $\delta \omega^{1-\gamma / 2}$ is bounded, we expect that the analysis performed by Ammari, Vogelius et al. (see for example [1], [5], [3], [2] or [10]) could be applied. Actually, in this case $\delta$ might be replaced by $c \times \omega^{-1+\gamma / 2}$, for an appropriate constant $c>0$, and only one parameter appears in our problem.

Theorem 1.2 (Main result). Let $\gamma \in[0,1]$.

Denote by $\Phi$ the $\mathscr{C}^{\infty}$-diffeomorphism, which maps a neighborhood of the cylinder $[0,1] \times \mathbb{T}$ unto a neighborhood of $\mathcal{O}_{\delta}$. Let $g \in H^{1 / 2}\left(\partial \Omega_{\delta}\right)$, and define $\mathfrak{g} \in H^{1 / 2}(\partial \mathcal{O})$ as:

$$
\mathfrak{g}=\left(\left.g \circ \Phi\right|_{\eta=1}\right) \circ\left(\left.\Phi\right|_{\eta=0}\right)^{-1} .
$$

Let $E$ be the solution in $H^{1}\left(\Omega_{\delta}\right)$ to problem (2), and let $v$ be the solution to the following problem:

$$
\begin{aligned}
& \nabla \cdot\left(\frac{1}{\mu_{c}} \nabla v\right)+\omega^{2} \widetilde{\varepsilon}_{c} v=0, \text { in } \mathcal{O} \\
& \left.\frac{\mu_{m}}{\mu_{c}} \partial_{n} v\right|_{\partial \mathcal{O}}-\left.\mathrm{A} v\right|_{\partial \mathcal{O}}=\mathrm{Bg}, \text { on } \partial \mathcal{O} .
\end{aligned}
$$

Then, the above function $v$ exists and is unique; it belongs to $H^{1}(\mathcal{O})$. Moreover, there exists $C>0$ such that for all $\omega \geq 1$, for all $\delta \in[0,1]$ such that $\delta \omega^{1-\gamma / 2}>C_{0}$, 
the following estimates hold:

$$
\|E-v\|_{L^{2}(\mathcal{O})} \leq c \omega^{\frac{5 \gamma-4}{2}} \sqrt{\delta}|\mathfrak{g}|_{H^{1 / 2}(\partial \mathcal{O})} .
$$

In addition, if $\gamma \in(4 / 5,1]$ and if $\omega$ is such that there exists a constant $c>0$ such that for all $k \in \mathbb{Z}$,

$$
\left|k^{2} / \omega^{2}-a_{m}\right| \geq c \omega^{-1 / 2}
$$

then

$$
\|E-v\|_{L^{2}(\mathcal{O})} \leq c \omega^{\gamma / 2-1} \sqrt{\delta}|\mathfrak{g}|_{H^{1 / 2}(\partial \mathcal{O})}
$$

Remark 1.3. Generalized impedance boundary condition (8) is a generalization of the Leontovitch condition. Actually, observe that if we consider few Fourier modes $(|k| \ll \omega)$ and if $\Re(\delta d)$ tends to $-\infty$, which means that we deal with very high frequencies (or equivalently, that $\delta$ does not tend to zero) we have:

$$
\begin{aligned}
& \mathfrak{s} \rightarrow 0, \\
& d \rightarrow \omega \sqrt{-q_{m}} .
\end{aligned}
$$

Hence (8) becomes:

$$
\left.\frac{\mu_{m}}{\mu_{c}} \partial_{n} v\right|_{\partial \mathcal{O}}-\left.\mathrm{i} \omega \sqrt{q_{m}} v\right|_{\partial \mathcal{O}}=0,
$$

which is exactly the Leontovitch impedance boundary condition (4).

Remark 1.4. Unfortunately, if $\gamma>4 / 5$ estimate (9) does not predict $L^{2}$-convergence for the full range of $\omega$ and $\delta$ we consider. In particular, for $\gamma=1$, the term $\delta \omega^{(5 \gamma-4) / 2}$ is greater than $C_{0}>0$, in accordance with hypothesis 1.1. Choosing the frequencies satisfying (10), we prove the convergence, however we think that too many frequencies are omitted in this condition and a weaker hypothesis should exist. This is a key-point, which we give our full attention at this time, and which is still an open problem as far as we know.

1.3. Numerical simulations. To illustrate the accuracy of our generalized impedance boundary condition, we compute the Fourier coefficients of $v$ and $E$ for a trigonometric polynomial boundary data $g$. To simplify computations, we approximate the above symbol $\mathfrak{s}$, which involves an integration of $d$ and $\widetilde{d}$. Actually, as we see later on, according to hypothesis 1.1 we have the following estimates:

$$
d=\widetilde{d}_{1}+O(\delta), \quad \widetilde{d}=\widetilde{d}_{1}+O(\delta) .
$$

We choose a trigonometric polynomial of degree 10, a thickness $\delta$ of order $10^{-2}$ and a pulsation $\omega$ of order $10^{3}$, therefore $\widetilde{d}_{1}(\omega, \eta, k) \sim \widetilde{d}_{1}(\omega, 0, k)$ and

$$
\int_{\eta}^{1} d(\omega, s, k) \mathrm{d} s \sim(1-\eta) d_{1}(\omega, 0, k) .
$$

Denote by $\mathscr{A}^{c o m p}$ and $\mathscr{B}^{c o m p}$ the operators used for the computations, defined by their respective symbols $s_{\mathscr{A}}^{c o m p}$ and $s_{\mathscr{B}}^{\text {comp }}$ :

$$
s_{\mathscr{A}}^{c o m p}=\widetilde{d}_{1}(\omega, 0, k)\left(1-2 \frac{e^{2 \delta \widetilde{d_{1}}(\omega, 0, k)}}{e^{2 \delta \widetilde{d_{1}}(\omega, 0, k)}-1}\right), \quad s_{\mathscr{B}}^{c o m p}=2 \frac{e^{\delta \widetilde{d_{1}}(\omega, 0, k)}}{e^{2 \delta \widetilde{d_{1}}(\omega, 0, k)}-1} .
$$


Let $\mathfrak{g}$ be the following trigonometric polynomial:

$$
\mathfrak{g}=\sum_{k=0}^{10} e^{\mathrm{i} k \theta}
$$

and let $\omega=800, \delta=10^{-2}, \gamma=2 / 3, q_{c}=1+\mathrm{i}$ and $q_{m}=2+2 \mathrm{i} / \omega^{\gamma}$. We compute the electric field $E$ defined by (2) and the solution $v$ to the following problem:

$$
\begin{aligned}
& \nabla \cdot\left(\frac{1}{\mu_{c}} \nabla v\right)+\omega^{2} \widetilde{\varepsilon}_{c} v=0, \text { in } \mathcal{O}, \\
& \left.\partial_{R} v\right|_{R=1}-\left.\mathscr{A}^{c o m p} v\right|_{R=1}=\mathscr{B}^{c o m p} \mathfrak{g} .
\end{aligned}
$$

We plot these two fields in Fig. 2 and Fig. 3. As we can see, the accuracy of our approximation in very satisfactory.

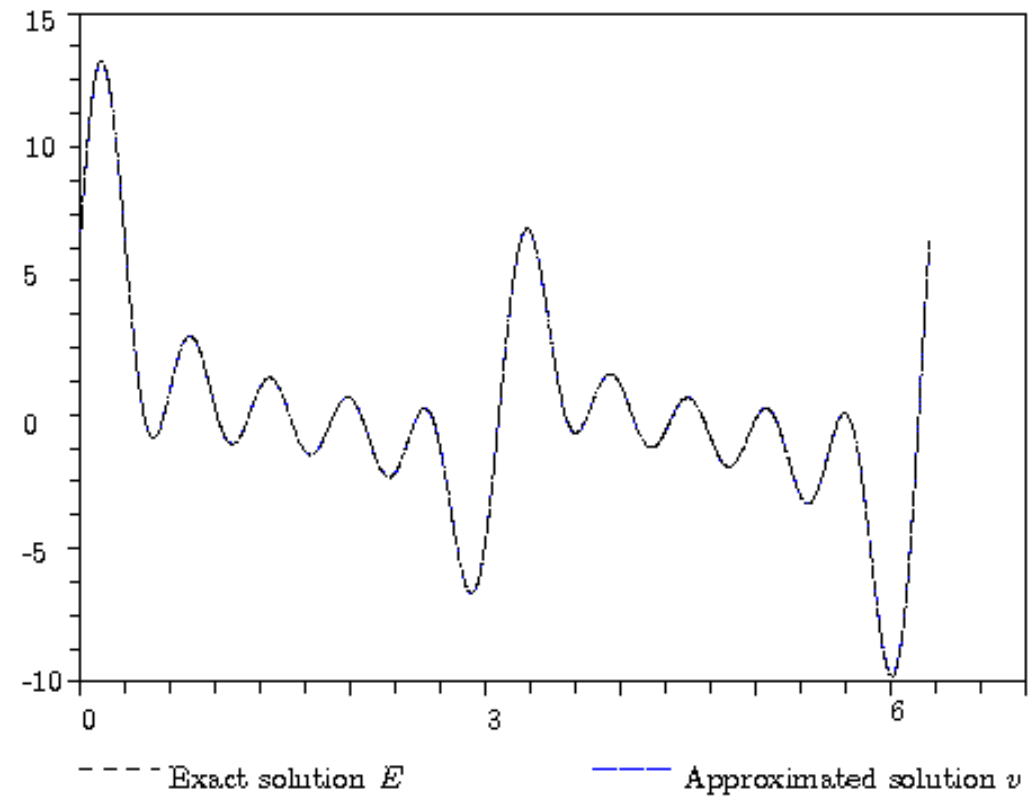

Figure 2. Traces of the fields $E$ and $v$ for $\theta \in(0,2 \pi)$.

A forthcoming work will deal with generalized boundary condition for a domain $\mathcal{O}$ of arbitrary shape. The microlocal analysis is quiet more difficult, in particular the derivatives of the curvature of $\partial \mathcal{O}$ lead to non trivial difficulties in the pseudodifferential calculus. Moreover, to perform numerical simulations it will be necessary to approximate the pseudodifferential operators A and B with differential operators. For all these reasons, we first choose to deal with the circular case. Observe also that with the help of Bessel-Parseval theorem, the circular case give Sobolev precise estimates on the errors, which are difficult to obtain in a more general geometry.

Let us now present the construction of our generalized impedance boundary condition, and let us prove our main result. 


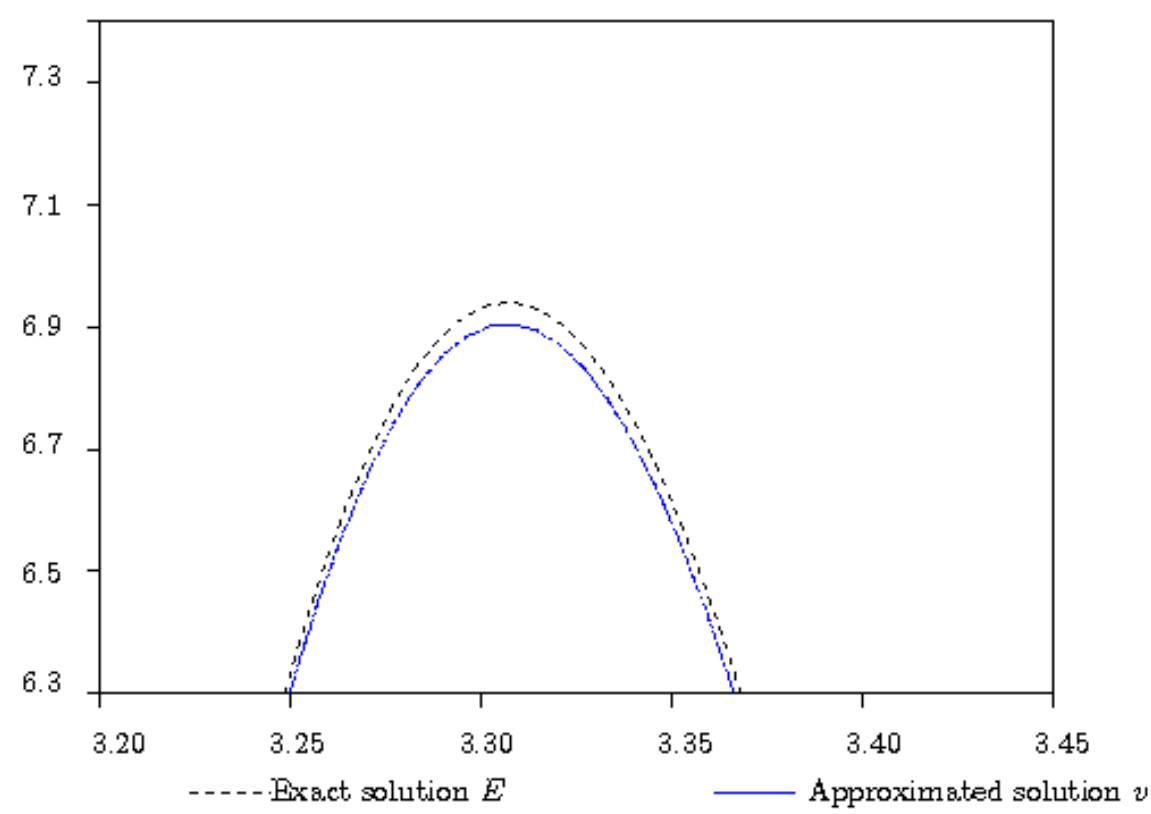

Figure 3. Zoom of Fig. 2 for $\theta \in(3.20,3.45)$.

\section{Factorization of the Helmholtz operator in the thin layeR}

Recall that $q_{m}=a_{m}+\mathrm{i} b_{m} \omega^{-\gamma}$, with $a_{m}>0, b_{m}>0$ and $\gamma \in[0,1]$, and denote by $\mathscr{L}$ Helmholtz operator in the thin layer:

$$
\mathscr{L}=\Delta+q_{m} \omega^{2} .
$$

Denote by $\boldsymbol{C}$ the cylinder $[0,1] \times \mathbb{T}$. It is well-known that $\mathscr{L}$ written in the rescaled polar coordinates $(\eta, \theta) \in \boldsymbol{C}$ equals:

$$
\mathscr{L}=\frac{1}{\delta^{2}} \partial_{\eta}^{2}+\frac{1}{(1+\delta \eta)^{2}} \partial_{\theta}^{2}+q_{m} \omega^{2}+\frac{1}{1+\delta \eta} \frac{1}{\delta} \partial_{\eta} .
$$

Our goal is to factorize $\mathscr{L}$ as follows:

$$
\mathscr{L}=\left(\frac{1}{\delta} \partial_{\eta}+\widetilde{D}\right)\left(\frac{1}{\delta} \partial_{\eta}-D\right)+R_{0}
$$

where $R_{0}$ is a bounded linear operator from $L^{2}(\mathbb{T})$ unto $L^{2}(\mathbb{T})$. The symbol $\sigma_{\mathscr{L}}$ of $\mathscr{L}$ equals:

$$
\begin{gathered}
\forall \omega \geq 1, \forall(\eta, \tau, k) \in[0,1] \times \mathbb{R} \times \mathbb{Z} \\
\sigma_{\mathscr{L}}(\omega, \eta, \tau, k)=-\frac{\tau^{2}}{\delta^{2}}-\frac{k^{2}}{(1+\delta \eta)^{2}}+q_{m} \omega^{2}+\frac{1}{1+\delta \eta} \frac{\mathrm{i} \tau}{\delta} .
\end{gathered}
$$

We exhibit the operators $D$ and $\widetilde{D}$ with the help of a well-known inductive process (see for instance Lafitte and Lebeau [7], Lafitte [8], Carvalho dos Santos and Hounie 
[4] or Vogelius et al. [6]). Define $d_{1}$ for all $(\omega, \eta, k) \in[1,+\infty[\times[0,1] \times \mathbb{Z}$ by:

$$
\left\{\begin{array}{l}
d_{1}(\omega, \eta, k)=\sqrt{\frac{k^{2}}{(1+\delta \eta)^{2}}-q_{m} \omega^{2}}, \\
\Re\left(d_{1}(\omega, \eta, k)\right) \leq 0 .
\end{array}\right.
$$

Observe that since $\Im\left(q_{m}\right)=b_{m} \omega^{-\gamma}$, there exists two positive constants $c$ and $C$ such that the following estimates hold for all $(\omega, \eta, k)$ in $[1,+\infty[\times[0,1] \times \mathbb{Z}$ :

$$
\begin{array}{rlrl}
\text { (14a) } & c(1+|k|+\omega) \omega^{-\gamma / 2} & \leq\left|d_{1}(\omega, \eta, k)\right| & \leq C(1+|k|+\omega), \\
\text { (14b) } & -C(1+|k|+\omega) & \leq \Re\left(d_{1}\right)(\omega, \eta, k) \leq-c(1+|k|+\omega) \omega^{-\gamma / 2} \leq 0, \\
(14 \mathrm{c}) & 0 \leq c(1+|k|+\omega) \omega^{-\gamma / 2} & \leq \Im\left(d_{1}\right)(\omega, \eta, k) \leq C(1+|k|+\omega) .
\end{array}
$$

Let $D_{1}$ be defined by its symbol $d_{1}$. To simplify notations, we write $D_{1}=O p\left(d_{1}\right)$; this means that for all $u$ in $\mathscr{C}^{\infty}(\boldsymbol{C})$, then

$$
\forall(\eta, \theta) \in C, \quad D_{1} u(\eta, \theta)=\frac{1}{2 \pi} \sum_{k \in \mathbb{Z}} d_{1}(\omega, \eta, k) \int_{0}^{2 \pi} u(\eta, t) e^{\mathrm{i} k(\theta-s)} \mathrm{d} s .
$$

First order factorization. Let us define $R_{1}$ as:

$$
\begin{aligned}
R_{1} & =\mathscr{L}-\left(\frac{1}{\delta} \partial_{\eta}+D_{1}\right)\left(\frac{1}{\delta} \partial_{\eta}-D_{1}\right), \\
& =\mathscr{L}-\left(\frac{1}{\delta^{2}} \partial_{\eta}^{2}-\frac{1}{\delta}\left[\partial_{\eta}, D_{1}\right]-D_{1}^{2}\right) .
\end{aligned}
$$

The symbol $\sigma_{R_{1}}$ of $R_{1}$ satisfies:

$$
\sigma_{R_{1}}=\frac{1}{1+\delta \eta} \frac{\mathrm{i} \tau}{\delta}+\frac{1}{\delta} \partial_{\eta} d_{1}
$$

Observe that

$$
\partial_{\eta} d_{1}(\omega, \eta, k)=\frac{-\delta k^{2}}{(1+\delta \eta)^{3} d_{1}(\omega, \eta, k)} .
$$

Second order factorization. Now, we find two operators $D_{0}$ and $\widetilde{D}_{0}$ such that

$$
R_{0}=\mathscr{L}-\left(\frac{1}{\delta} \partial_{\eta}+\left(D_{1}+\widetilde{D}_{0}\right)\right)\left(\frac{1}{\delta} \partial_{\eta}-\left(D_{1}+D_{0}\right)\right),
$$

is an operator of lower order than $R_{1}$. Observe that

$$
\begin{aligned}
R_{0} & =R_{1}+\left(D_{0}+\widetilde{D}_{0}\right) D_{1}+\frac{1}{\delta}\left(D_{0}-\widetilde{D}_{0}\right) \partial_{\eta} \\
& +\left[D_{1}, D_{0}\right]+\frac{1}{\delta}\left[\partial_{\eta}, D_{0}\right]+\widetilde{D}_{0} D_{0} .
\end{aligned}
$$

We denote by $d_{0}$ and $\widetilde{d}_{0}$ the respective symbols of $D_{0}$ and $\widetilde{D}_{0}$. Define:

$$
\begin{aligned}
& d_{0}-\widetilde{d}_{0}=-\frac{1}{1+\delta \eta}, \\
& d_{0}+\widetilde{d}_{0}=-\frac{\partial_{\eta} d_{1}}{\delta d_{1}} .
\end{aligned}
$$


Therefore, we have

$$
\begin{aligned}
& \widetilde{d}_{0}=-\frac{\partial_{\eta} d_{1}}{2 \delta d_{1}}+\frac{1}{2(1+\delta \eta)} \\
& d_{0}=-\frac{\partial_{\eta} d_{1}}{2 \delta d_{1}}-\frac{1}{2(1+\delta \eta)}
\end{aligned}
$$

Accordingly (15),

$$
\frac{\partial_{\eta} d_{1}}{2 \delta d_{1}}=-\frac{k^{2}}{(1+\delta \eta)^{3} d_{1}^{2}}
$$

hence estimate (14) implies there exists a positive constants $C$

$$
\left|\widetilde{d}_{0}\right|,\left|d_{0}\right| \leq C \omega^{\gamma}
$$

moreover, if $k^{2} / \omega^{2} \sim a_{m}$, the symbols $\widetilde{d}_{0}$ and $d_{0}$ are exactly of order $\omega^{\gamma}$. Define $d$ and $\widetilde{d}$ as follows

$$
\begin{aligned}
& d(\omega, \eta, k)=d_{1}(\omega, \eta, k)+d_{0}(\omega, \eta, k), \\
& \widetilde{d}(\omega, \eta, k)=d_{1}(\omega, \eta, k)+\widetilde{d}_{0}(\omega, \eta, k),
\end{aligned}
$$

and let :

$$
D=\mathrm{Op}(d), \quad \widetilde{D}=\mathrm{Op}(\widetilde{d})
$$

Observe that

$$
\begin{aligned}
& d(\omega, \eta, k)=d_{1}(\omega, \eta, k)+\frac{k^{2}}{2(1+\delta \eta)^{3} d_{1}^{2}}+\frac{1}{2(1+\delta \eta)} \\
& \widetilde{d}(\omega, \eta, k)=d_{1}(\omega, \eta, k)+\frac{k^{2}}{2(1+\delta \eta)^{3} d_{1}^{2}}-\frac{1}{2(1+\delta \eta)}
\end{aligned}
$$

If $k^{2} / \omega^{2} \sim a_{m}$, the term $k^{2} /\left(2(1+\delta \eta)^{3} d_{1}^{2}\right)$ is of order $\omega^{\gamma}$, whereas $d_{1}$ is similar to $\omega^{1-\gamma / 2}$. Therefore, as soon as $\gamma$ belongs to $(2 / 3,1]$, if $k^{2} / \omega^{2} \sim a_{m}$ we have:

$$
\left|\frac{\partial_{\eta} d_{1}(\omega, \eta, k)}{\delta d_{1}(\omega, \eta, k)}\right|>\left|d_{1}(\omega, \eta, k)\right|
$$

which means that $d_{1}$ is not exactly the leading symbol of $d$ and $\widetilde{d}$. Define

$$
\widetilde{d}_{1}(\omega, \eta, k)=d_{1}(\omega, \eta, k)-\frac{\partial_{\eta} d_{1}(\omega, \eta, k)}{2 \delta d_{1}(\omega, \eta, k)},
$$

we have

$$
\begin{aligned}
d(\omega, \eta, k) & =\widetilde{d}_{1}(\omega, \eta, k)+O(1), \\
\widetilde{d}(\omega, \eta, k) & =\widetilde{d}_{1}(\omega, \eta, k)+O(1) .
\end{aligned}
$$

A simple calculation implies that for all $\omega \geq 1$, for all $\delta \in(0,1)$ and for all $k \in \mathbb{Z}$ :

$$
\begin{aligned}
& c\left((1+|k|+\omega) \omega^{-\gamma / 2}+\omega^{\gamma}\right) \leq|d(\omega, \eta, k)| \leq C(1+|k|+\omega), \\
& c\left((1+|k|+\omega) \omega^{-\gamma / 2}+\omega^{\gamma}\right) \leq|\widetilde{d}(\omega, \eta, k)| \leq C(1+|k|+\omega),
\end{aligned}
$$

Therefore, we have factorized the operator $\mathscr{L}$ :

$$
\mathscr{L}=\left(\frac{1}{\delta} \partial_{\eta}+D_{1}+D_{0}\right)\left(\frac{1}{\delta} \partial_{\eta}-D_{1}-\widetilde{D}_{0}\right)+R_{0} .
$$


Since we deal with the circular case, the commutator $\left[D_{1}, D_{0}\right]$ of the two pseudodifferential operators $D_{1}$ and $D_{0}$ equals zero. In vertue of (16), the symbol $\sigma_{R_{0}}$ equals

$$
\sigma_{R_{0}}=\frac{1}{\delta} \partial_{\eta} d_{0}+\widetilde{d}_{0} d_{0}
$$

Therefore, according to (18) there exists a constant $C$ such that for all $\eta \in[0,1]$, for all $\omega \geq 1$, for all $\delta \in(0,1)$, for all $k \in \mathbb{Z}$, the following estimate holds:

$$
\left|\sigma_{R_{0}}(\omega, \eta, k)\right| \leq C \omega^{2 \gamma} .
$$

Moreover, for $k^{2} / \omega^{2} \sim a_{m}, \sigma_{R_{0}}$ is exactly of order $\omega^{2 \gamma}$. Therefore $R_{0}$ is a pseudodifferential operator on the torus of order 0 but as soon as $\gamma \neq 0$ it is not of order 0 in the $\omega$-calculus of Lebeau and Lafitte since its symbol is bounded by $\omega^{2 \gamma}$ and not by an $\omega$-independent constant.

If we omit some values of $\omega$, we may obtain a better estimate of $\sigma_{R_{0}}$, which will be useful to prove estimate (11). Actually, using the definitions the symbols $d_{1}, d_{0}$ and $\widetilde{d}_{0}$, the symbol $\sigma_{R_{0}}$ equals:

$$
\begin{aligned}
\sigma_{R_{0}} & =-\frac{\partial_{\eta}^{2} d_{1}}{2 \delta^{2} d_{1}}+\frac{3\left(\partial_{\eta} d_{1}\right)^{2}}{4 \delta^{2} d_{1}^{2}}+\frac{1}{4(1+\delta \eta)^{2}} \\
& =\frac{4 q_{m} k^{2} / \omega^{2}+q_{m}^{2}(1+\delta \eta)^{2}}{4\left(k^{2} / \omega^{2}-q_{m}(1+\delta \eta)^{2}\right)^{2}} .
\end{aligned}
$$

Hence, if we choose $\omega$ such that there exists a constant $c>0$ such that for all $k \in \mathbb{Z}$,

$$
\left|k^{2} / \omega^{2}-a_{m}\right| \geq c \omega^{-1 / 2},
$$

the following estimate of $\sigma_{R_{0}}$ holds:

$$
\left|\sigma_{R_{0}}(\omega, \eta, k)\right| \leq C \omega .
$$

\section{THE FACTORIZED PROBLEM}

Let us study the factorized problem in the thin layer. Suppose that we know the traces of the electric field on the boundaries of the layer and denote it by $\mathfrak{g}$ in $\eta=1$ and $h_{0}$ in $\eta=0$. We consider the solution $(\widehat{v}, \widehat{\gamma})$ to the following problem, for all $k \in \mathbb{Z}$ :

$$
\begin{aligned}
& \frac{1}{\delta} \partial_{\eta} \widehat{v}(\eta, k)-d(\omega, \eta, k) \widehat{v}(\eta, k)=\widehat{\gamma}(\eta, k), \\
& \frac{1}{\delta} \partial_{\eta} \widehat{\gamma}(\eta, k)+\widetilde{d}(\omega, \eta, k) \widehat{\gamma}(\eta, k)=0
\end{aligned}
$$

with Dirichlet boundary conditions

$$
\widehat{v}(0, k)=\widehat{h_{0}}(k), \quad \widehat{v}(1, k)=\widehat{\mathfrak{g}}(k) .
$$

A direct computation of the Duhamel rule implies:

$$
\begin{aligned}
& \widehat{v}(\eta, k)=e^{\delta \int_{0}^{\eta} d(\omega, s, k) \mathrm{d} s} \widehat{h_{0}}(k)+\delta \int_{0}^{\eta} e^{\delta \int_{\eta^{\prime}}^{\eta} d(\omega, s, k) \mathrm{d} s} \widehat{\gamma}\left(\eta^{\prime}, k\right) \mathrm{d} \eta^{\prime}, \\
& \widehat{\gamma}(\eta, k)=e^{\delta \int_{\eta}^{1} \widetilde{d}(\omega, s, k) \mathrm{d} s} \widehat{\gamma}(1, k),
\end{aligned}
$$


and $\widehat{\gamma}(1, k)$ is defined by:

$$
\left(\int_{0}^{1} e^{\delta \int_{\eta}^{1}(d+\widetilde{d})(\omega, s, k) \mathrm{d} s} \mathrm{~d} \eta\right) \widehat{\gamma}(1, k)=\frac{1}{\delta}\left(\widehat{\mathfrak{g}}(k)-e^{\delta \int_{0}^{1} d(\omega, s, k) \mathrm{d} s} \widehat{h_{0}}(k)\right) .
$$

We have to prove

$$
\int_{0}^{1} e^{\delta \int_{\eta^{\prime}}^{1}(d+\widetilde{d})(\omega, s, k) \mathrm{d} s} \mathrm{~d} \eta^{\prime} \neq 0,
$$

so that $\widehat{v}$ might be uniquely determined. According to (21),

$$
\delta(d+\widetilde{d})(\omega, s, k)=2 \delta \widetilde{d}_{1}(\omega, s, k)+O(\delta) .
$$

Therefore

$$
\int_{0}^{1} e^{\delta \int_{\eta^{\prime}}^{1}(d+\widetilde{d})(\omega, s, k) \mathrm{d} s} \mathrm{~d} \eta^{\prime}=\int_{0}^{1} e^{2 \delta \int_{\eta^{\prime}}^{1} \widetilde{d_{1}}(\omega, s, k) \mathrm{d} s} \mathrm{~d} \eta^{\prime}(1+O(\delta)) .
$$

Accordingly (20)

$$
2 \delta \int_{\eta^{\prime}}^{1} \widetilde{d}_{1}(\omega, s, k) \mathrm{d} s=2 \delta \int_{\eta^{\prime}}^{1} d_{1}(\omega, s, k) \mathrm{d} s+\log \left(\frac{d_{1}\left(\omega, \eta^{\prime}, k\right)}{d_{1}(\omega, 1, k)}\right),
$$

hence we infer

$$
\int_{0}^{1} e^{2 \delta \int_{\eta^{\prime}}^{1} \widetilde{d_{1}}(\omega, s, k) \mathrm{d} s} \mathrm{~d} \eta^{\prime}=\frac{1}{2 \delta d_{1}(\omega, 1, k)}\left(e^{2 \delta \int_{0}^{1} d_{1}(\omega, s, k) \mathrm{d} s}-1\right) .
$$

Therefore, since $\Re\left(d_{1}\right)$ never vanishes, (31) holds and we may define by $\mathfrak{p}$ and $\mathfrak{s}$ the two following symbols, for all $\omega \geq \omega_{0}$, for all integer $k \in \mathbb{Z}$ :

$$
\begin{aligned}
& \mathfrak{p}(\omega, k)=\left(\int_{0}^{1} e^{\delta \int_{x^{\prime}}^{1}(d+\widetilde{d})(\omega, s, k) \mathrm{d} s} \mathrm{~d} x^{\prime}\right)^{-1}, \\
& \mathfrak{s}(\omega, k)=\frac{1}{\delta} \mathfrak{p}(\omega, k) e^{\delta \int_{0}^{1} \widetilde{d}(\omega, s, k) \mathrm{d} s} .
\end{aligned}
$$

Using (28)-(30) we obtain the following equality:

$$
\frac{1}{\delta} \partial_{\eta} \widehat{v}(0, k)-\left(d(\omega, 0, k)-\mathfrak{s}(\omega, k) e^{\delta \int_{0}^{1} d(\omega, s, k) \mathrm{d} s}\right) \widehat{h_{0}}(k)=\mathfrak{s}(\omega, k) \widehat{\mathfrak{g}}(k) .
$$

Denote by $\mathscr{A}$ and $\mathscr{B}$ the two following pseudodifferential operators:

$$
\begin{aligned}
& \forall \theta \in \mathbb{T}, \forall u \in \mathscr{C}^{\infty}(\mathbb{T}), \\
& \mathscr{A} u(\theta)=\frac{1}{2 \pi} \sum_{k \in \mathbb{Z}}\left(d(\omega, 0, k)-\mathfrak{s}(\omega, k) e^{\delta \int_{0}^{1} d(\omega, s, k) \mathrm{d} s}\right) \int_{0}^{2 \pi} u(s) e^{\mathrm{i} k(t-s)} \mathrm{d} s, \\
& \mathscr{B} u(\theta)=\frac{1}{2 \pi} \sum_{k \in \mathbb{Z}} \mathfrak{s}(\omega, k) \int_{0}^{2 \pi} u(s) e^{\mathrm{i} k(t-s)} \mathrm{d} s,
\end{aligned}
$$

and let $\sigma_{\mathscr{A}}$ and $\sigma_{\mathscr{B}}$ be the respective symbols of $\mathscr{A}$ and $\mathscr{B}$. Observe that

$$
\begin{aligned}
\sigma_{\mathscr{A}} & =d(\omega, 0, k)-\mathfrak{s}(\omega, k) e^{\delta \int_{0}^{1} d(\omega, s, k) \mathrm{d} s}, \\
& =\left.d_{1}\right|_{\eta=0}\left(1-2 \frac{e^{2 \delta \int_{0}^{1} d_{1}(\omega, s, k) \mathrm{d} s}}{e^{2 \delta \int_{0}^{1} d_{1}(\omega, s, k) \mathrm{d} s}-1}\right)(1+O(\delta))-\frac{\left.\partial_{\eta} d_{1}\right|_{\eta=0}}{\left.2 \delta d_{1}\right|_{\eta=0}},
\end{aligned}
$$


and

$$
\sigma_{\mathscr{B}}=\frac{1}{\delta} \frac{e^{\delta \int_{0}^{1} d_{1}(\omega, s, k) \mathrm{d} s}}{e^{2 \delta \int_{0}^{1} d_{1}(\omega, s, k) \mathrm{d} s}-1}(1+O(\delta)) .
$$

We suppose that

$$
\delta \omega^{1-\gamma / 2} \geq C_{0}
$$

with $C_{0}$ such that

$$
\left|\frac{e^{2 \delta \int_{0}^{1} d_{1}(\omega, s, k) \mathrm{d} s}}{e^{2 \delta \int_{0}^{1} d_{1}(\omega, s, k) \mathrm{d} s}-1}\right|<\frac{1}{2} .
$$

Therefore, there exists a constant $c$ such that for all $k \in \mathbb{Z}$, for all $\omega \geq 1$, for all $\delta \omega^{1-\gamma / 2} \geq C_{0}$, for all $\eta \in(0,1)$,

$$
\Im\left(\sigma_{\mathscr{A}}(\eta, \omega, k)\right) \geq c\left((1+|k|+\omega) \omega^{-\gamma / 2}+\omega^{\gamma}\right),
$$

and

$$
\left|\sigma_{\mathscr{B}}(\eta, \omega, k)\right| \leq \frac{C e^{-c \delta \omega^{1-\gamma / 2}}}{\delta} .
$$

Remark 3.1. For fixed $\delta$ and $\omega$ since $\sigma_{\mathscr{B}}$ decreases exponentially, $\mathscr{B}$ is a regularizing operator in the pseudodifferential calculus on the torus, however we cannot obtain a bound uniform in $\omega$ for this regularizing operator for $\omega$ tending to infinity and $\delta$ tending to zero, with $\delta \omega^{1-\gamma / 2} \geq C_{0}$. The estimate (39) gives a uniform bound of $\sigma_{\mathscr{B}}$ in terms of such $\omega$ and $\delta$, but we loose the regularizing aspect of $\mathscr{B}$.

Equality (36) and the definitions of pseudodifferential operators $\mathscr{A}$ and $\mathscr{B}$ lead to the impedance boundary condition in $\eta=0$ :

$$
\left.\frac{1}{\delta} \partial_{\eta} v\right|_{\eta=0}-\left.\mathscr{A} v\right|_{\eta=0}=\mathscr{B} \mathfrak{g} .
$$

The following lemma will be very useful.

Lemma 3.2. Let $\mathfrak{g}$ and $h_{0}$ be in $H^{s}(\mathbb{T})$, for $s \geq 0$. Let $\gamma \in[0,1]$, and let $C_{0}$ defined by (37). There exists a constant $C$ such that the solution $v$ to the Cauchy problem:

$$
\left(\frac{1}{\delta} \partial_{\eta} v+\widetilde{D}(\omega, \eta)\right)\left(\frac{1}{\delta} \partial_{\eta} v-D(\omega, \eta)\right) v=0,
$$

with Dirichlet boundary conditions

$$
\left.v\right|_{\eta=0}=h_{0},\left.\quad v\right|_{\eta=1}=\mathfrak{g} .
$$

satisfies the following estimate, for all $\omega \geq 1$ and for all $\delta \in(0,1)$ such that $\delta \omega^{1-\gamma / 2} \geq C_{0}$ :

$$
\|v\|_{\Lambda^{0} L^{2}(\boldsymbol{C})} \leq C \sqrt{\delta}\left(\left\|h_{0}\right\|_{H^{s}(\mathbb{T})}+\|\mathfrak{g}\|_{H^{s}(\mathbb{T})}\right),
$$

where

$$
\|v\|_{\Lambda^{0} L^{2}(\boldsymbol{C})}^{2}=\int_{0}^{1} \int_{0}^{2 \pi} \delta(1+\delta \eta)|v(\eta, \theta)|^{2} \mathrm{~d} \eta \mathrm{d} \theta .
$$


Remark 3.3. Denote by $\Phi$ the $\mathscr{C}^{\infty}$-diffeomorphism, which maps a neighborhood of $\boldsymbol{C}$ unto a neighborhood of $\mathcal{O}_{\delta}$. Then we have, for $z \in L^{2}\left(\mathcal{O}_{\delta}\right)$,

$$
\|z\|_{L^{2}\left(\mathcal{O}_{\delta}\right)}=\|z \mathrm{O} \Phi \mathrm{O}\|_{\Lambda^{0} L^{2}(\boldsymbol{C})} .
$$

Proof. We prove this lemma using Fourier coefficients of $v$. According to (29), for all $k \in \mathbb{Z}, \widehat{v}_{k}$ is given by

$$
\begin{aligned}
\widehat{v}(\eta, k) & =\left(\int_{0}^{\eta} e^{\delta \int_{\eta^{\prime}}^{\eta} d(\omega, s, k) \mathrm{d} s} e^{\delta \int_{\eta^{\prime}}^{1} \widetilde{d}(\omega, s, k) \mathrm{d} s} \mathrm{~d} \eta^{\prime}\right) \mathfrak{p}(\omega, k) \\
& \times\left(\widehat{\mathfrak{g}}(k)-e^{\delta \int_{0}^{1} d(\omega, s, k) \mathrm{d} s} \widehat{h_{0}}(k)\right)+e^{\delta \int_{0}^{\eta} d(\omega, s, k) \mathrm{d} s} \widehat{h_{0}}(k) .
\end{aligned}
$$

Using equalities (32) and (33), a simple calculation implies:

$$
e^{\delta \int_{0}^{\eta} d(\omega, s, k) \mathrm{d} s}=\sqrt{\frac{d_{1}(\omega, 0, k)}{d_{1}(\omega, \eta, k)}} e^{\delta \int_{0}^{\eta} d_{1}(\omega, s, k) \mathrm{d} s}(1+O(\delta))
$$

and

$$
\begin{aligned}
\mathfrak{p}(\omega, k) \int_{0}^{\eta} e^{\delta \int_{\eta^{\prime}}^{\eta} d(\omega, s, k) \mathrm{d} s} e^{\delta \int_{\eta^{\prime}}^{1} \widetilde{d}(\omega, s, k) \mathrm{d} s} \mathrm{~d} \eta^{\prime} & =\sqrt{\frac{d_{1}(\omega, 1, k)}{d_{1}(\omega, \eta, k)}} e^{\delta \int_{\eta}^{1} d_{1}(\omega, s, k) \mathrm{d} s} \\
& \times \frac{e^{2 \delta \int_{0}^{\eta} d_{1}(\omega, s, k) \mathrm{d} s}-1}{e^{2 \delta \int_{0}^{1} d_{1}(\omega, s, k) \mathrm{d} s}-1}(1+O(\delta)),
\end{aligned}
$$

Using (14b) and the definition of $C_{0}$, there exists a constant $C>0$ such that, for all $k \geq$, for all $\omega \geq 1$ and for all $\delta$ such that $\delta \omega^{1-\gamma / 2} \geq C_{0}$, we have for all $\eta \in[0,1]$ :

$$
|\widehat{v}(\eta, k)| \leq C\left(\left|\widehat{h_{0}}(k)\right|+|\widehat{\mathfrak{g}}(k)|\right),
$$

from which we infer (41), with the help of Bessel-Parseval equality.

\section{Generalized impedance boundary condition}

Let $\Psi$ be the $\mathscr{C}^{\infty}$-diffeomorphism, which maps the torus unto $\partial \mathcal{O}$. We denote by $\mathrm{A}, \mathrm{B}$ and $\mathrm{R}_{0}$ the three following pseudodifferential operators:

$$
\begin{aligned}
\forall s & \in \mathbb{R}, \forall u \in H^{s}(\partial \mathcal{O}), \\
\mathrm{A} u & =(\mathscr{A}(u \circ \Psi)) \circ \Psi^{-1}, \\
\mathrm{~B} u & =(\mathscr{B}(u \circ \Psi)) \circ \Psi^{-1}, \\
\mathrm{R}_{0} u & =\left(R_{0}(u \circ \Psi)\right) \circ \Psi^{-1} .
\end{aligned}
$$

Consider Helmholtz equation in the inner ball $\mathcal{O}$ with the generalized impedance condition deduced from (40). Using transmission conditions (3) and equality (40), we define $v$ in $\mathcal{O}$ by :

$$
\left\{\begin{array}{l}
\Delta v+\omega^{2} q_{c} v=0, \text { in } \mathcal{O}, \\
\left.\frac{\mu_{m}}{\mu_{c}} \partial_{n} v\right|_{\partial \mathcal{O}}-\left.\mathrm{A} v\right|_{\partial \mathcal{O}}=\mathrm{Bg} .
\end{array}\right.
$$

The generalized boundary condition imposed to $v$ should approach the influency of the thin membrane on the field of the inner domain $\mathcal{O}$. In this section, we estimate the error due to this approximation. 


\subsection{Existence and uniqueness.}

Lemma 4.1. Let $\gamma \in[0,1]$, and $q_{c}=a_{c}+\mathrm{i} b_{c}$, with $a_{c}$ and $b_{c}$ strictly positive constants.

For all $\mathfrak{g} \in L^{2}(\partial \mathcal{O})$, there exists an unique solution $v$ in $H^{1}(\mathcal{O})$ satisfying:

$$
\begin{aligned}
& \Delta v+\omega^{2} q_{c} v=0, \text { in } \mathcal{O}, \\
& \left.\frac{\mu_{m}}{\mu_{c}} \partial_{n} v\right|_{\partial \mathcal{O}}-\left.\mathrm{A} v\right|_{\partial \mathcal{O}}=\mathfrak{g}, \text { on } \partial \mathcal{O} .
\end{aligned}
$$

This solution $v$ satisfies, for all $\omega \geq 1$, for all $\delta \in(0,1)$ such that $\delta \omega^{1-\gamma / 2} \geq C_{0}$ the following estimates:

$$
\begin{aligned}
|v|_{L^{2}(\partial \mathcal{O})} & \leq C \frac{1}{\omega^{1-\gamma / 2}+\omega^{\gamma}}|\mathfrak{g}|_{L^{2}(\partial \mathcal{O})} \\
\|v\|_{L^{2}(\mathcal{O})} & \leq C \frac{1}{\omega\left(\omega^{1-\gamma / 2}+\omega^{\gamma}\right)}|\mathfrak{g}|_{L^{2}(\partial \mathcal{O})} .
\end{aligned}
$$

Proof. We prove uniqueness and existence of $v$ using Fourier analysis. If such $v$ exists and belongs to $H^{1}(\mathcal{O})$, its Fourier coefficient $\widehat{v}(\cdot, k)$ satisfies for $k \in \mathbb{Z}$ :

$$
\begin{aligned}
& \frac{1}{r} \frac{d}{d r}\left(r \frac{d}{d r} \widehat{v}(r, k)\right)+\left(\omega^{2} q_{c}-k^{2}\right) \widehat{v}(r, k)=0, \text { for } r \in[0,1], \\
& \frac{\mu_{m}}{\mu_{c}} \frac{d}{d r} \widehat{v}(1, k)-\sigma_{\mathscr{A}}(\omega, k) \widehat{v}(1, k)=\widehat{\mathfrak{g}}(k) .
\end{aligned}
$$

- Uniqueness and existence. Let $\mathfrak{g}$ equal zero. By multiplying (47) by $r \overline{\widehat{v}}(r, k)$, by integrating by parts and taking the imaginary part, we infer:

$$
b_{c} \omega^{2} \int_{0}^{1} r|\widehat{v}(r, k)|^{2} \mathrm{~d} r+\frac{\mu_{c}}{\mu_{m}} \Im\left(\sigma_{\mathscr{A}}(\omega, k)\right)|\widehat{v}(1, k)|^{2}=0 .
$$

According to (38), we straight infer that $v$ vanishes identically. This implies directly the existence through a classical argument.

- Estimates. Let $\mathfrak{g}$ be in $L^{2}(\partial \mathcal{O})$. Once again, by multiplying $(47)$ by $r \overline{\hat{v}}(r, k)$, by integrating by parts and taking the imaginary part, we get:

$$
b_{c} \omega^{2} \int_{0}^{1} r|\widehat{v}(r, k)|^{2} \mathrm{~d} r+\frac{\mu_{c}}{\mu_{m}} \Im\left(\sigma_{\mathscr{A}}(\omega, k)\right)|\widehat{v}(1, k)|^{2}=\widehat{\mathfrak{g}}_{k} \overline{\widehat{v}}(1, k) .
$$

Estimate (38) implies there exists a constant $C>0$ such that for all $\omega \geq 1$, for all $\delta \in(0,1)$ such that $\delta \omega^{1-\gamma / 2} \geq C_{0}$, for all $k \in \mathbb{Z}$,

$$
|\widehat{v}(1, k)| \leq C \frac{1}{\omega^{1-\gamma / 2}+\omega^{\gamma}}\left|\widehat{\mathfrak{g}}_{k}\right|
$$

Estimates (46) follows straightforward.

4.2. Main result. We are now ready to prove our main result.

Theorem 4.2. Let $\gamma \in[0,1]$.

Let $g \in H^{1 / 2}\left(\partial \Omega_{\delta}\right)$. Let $\Phi$ the $\mathscr{C}^{\infty}$-diffeomorphism, which maps a neighborhood of $\boldsymbol{C}$ unto a neighborhood of the thin layer $\mathcal{O}_{\delta}$. Define

$$
\mathfrak{g}=\left.g \circ \Phi\right|_{\eta=1} \text {. }
$$


Let $\mu$ and $\widetilde{\varepsilon}$ be the following piecewise functions

$$
\mu=\left\{\begin{array}{l}
\mu_{c}, \text { in } \mathcal{O}, \\
\mu_{m}, \text { in } \mathcal{O}_{\delta},
\end{array} \quad \widetilde{\varepsilon}=\left\{\begin{array}{l}
\widetilde{\varepsilon}_{c}, \text { in } \mathcal{O}, \\
\widetilde{\varepsilon}_{m}, \text { in } \mathcal{O}_{\delta},
\end{array}\right.\right.
$$

and let $q_{c}=\mu_{c} \widetilde{\varepsilon}_{c}, q_{m}=\mu_{m} \widetilde{\varepsilon}_{m}$. We suppose that there exists $b_{c}$ and $b_{m}$ strictly positive constants such that $\Im\left(q_{m}\right)=b_{m} \omega^{-\gamma}$ and $\Im\left(q_{c}\right)=b_{c}$.

We denote by $E$ the solution to Helmholtz equation with Dirichlet boundary condition $g$ :

$$
\begin{aligned}
& \operatorname{div}\left(\frac{1}{\mu} \operatorname{grad} E\right)+\omega^{2} \widetilde{\varepsilon} E=0, \text { in } \Omega_{\delta}=\overline{\mathcal{O} \cup \mathcal{O}_{\delta}}, \\
& \left.E\right|_{\partial \Omega_{\delta}}=g \text {, on } \partial \Omega_{\delta} .
\end{aligned}
$$

Let $(u, v)$ be the solution to the following problem:

$$
\begin{aligned}
& \left(\partial_{\eta}+\delta \widetilde{D}\right)\left(\partial_{\eta}-\delta D\right) u=0, \text { for }(\eta, \theta) \in \boldsymbol{C}, \\
& \operatorname{div}\left(\frac{1}{\mu_{c}} \operatorname{grad} v\right)+\omega^{2} \widetilde{\varepsilon}_{c} v=0, \text { in } \mathcal{O},
\end{aligned}
$$

with transmission conditions :

$$
\begin{aligned}
& \left.\frac{1}{\mu_{c}} \partial_{n} v \circ \Phi\right|_{\eta=0}=\left.\frac{1}{\mu_{m}} \frac{1}{\delta} \partial_{\eta} u\right|_{\eta=0}, \\
& \left.v \circ \Phi\right|_{\eta=0}=\left.u\right|_{\eta=0},
\end{aligned}
$$

and the Dirichlet boundary condition

$$
\left.u\right|_{\eta=1}=\mathfrak{g} .
$$

Define

$$
v=\left\{\begin{array}{l}
v, \text { in } \mathcal{O}, \\
u \circ \Phi^{-1} \text { in } \mathcal{O}_{\delta} .
\end{array}\right.
$$

There exists $c>0$, such that for all $\omega \geq 1$, for all $\delta \in(0,1)$ such that $\delta \omega^{1-\gamma / 2} \geq C_{0}$ we have

$$
\|E-v\|_{L^{2}\left(\mathcal{O}_{\delta}\right)} \leq c \omega^{3 \gamma-2} \sqrt{\delta}|\mathfrak{g}|_{H^{1 / 2}(\mathbb{T})},
$$

and in $\mathcal{O}$ :

$$
\|E-v\|_{L^{2}(\mathcal{O})} \leq c \omega^{\frac{5 \gamma-4}{2}} \sqrt{\delta}|\mathfrak{g}|_{H^{1 / 2}(\mathbb{T})} .
$$

In addition, if $\gamma \in(2 / 3,1]$ and if $\omega$ is chosen such that there exists a constant $c>0$ such that for all $k \in \mathbb{Z}$,

$$
\left|k^{2} / \omega^{2}-a_{m}\right| \geq c \omega^{-1 / 2},
$$

then

$$
\|E-v\|_{L^{2}\left(\mathcal{O}_{\delta}\right)} \leq c \omega^{\gamma-1} \sqrt{\delta}|\mathfrak{g}|_{H^{1 / 2}(\mathbb{T})},
$$

and in $\mathcal{O}$ :

$$
\|E-v\|_{L^{2}(\mathcal{O})} \leq c \omega^{\gamma / 2-1} \sqrt{\delta}|\mathfrak{g}|_{H^{1 / 2}(\mathbb{T})}
$$


Remark 4.3. Observe that $v$ is the unique solution to the following problem:

$$
\begin{aligned}
& \Delta v+\omega^{2} q_{c} v=0, \text { in } \mathcal{O}, \\
& \left.\frac{\mu_{m}}{\mu_{c}} \partial_{n} v\right|_{\partial \mathcal{O}}-\left.\mathrm{A} v\right|_{\partial \mathcal{O}}=\operatorname{Bgo}\left(\left.\Phi\right|_{\eta=0}\right)^{-1}, \text { on } \partial \mathcal{O} .
\end{aligned}
$$

Hence this theorem gives estimates of the error perfomed by the generalized impedance boundary condition. Theorem 1.2 is a direct corollary of this theorem.

Proof. According to Lemma 4.1 and estimate (39), we infer:

$$
\left.|v|_{\partial \mathcal{O}}\right|_{L^{2}(\partial \mathcal{O})} \leq C|\mathfrak{g}|_{H^{1 / 2}(\mathbb{T})}
$$

Define $w=E-v$; it satisfies

$$
\begin{aligned}
& \Delta w+\omega^{2} q_{c} w=0, \text { in } \mathcal{O}, \\
& \Delta w+\omega^{2} q_{m} w=\mathrm{R}_{0} v, \text { in } \mathcal{O}_{\delta}, \\
& \left.w\right|_{\partial \Omega_{\delta}}=0, \text { on } \partial \Omega_{\delta},
\end{aligned}
$$

with transmission conditions

$$
\begin{aligned}
& \left.w\right|_{\partial \mathcal{O}^{+}}=\left.w\right|_{\partial \mathcal{O}^{-}}, \\
& \left.\mu_{m} \partial_{n} w\right|_{\partial \mathcal{O}^{+}}=\left.\mu_{c} \partial_{n} w\right|_{\partial \mathcal{O}^{-}} .
\end{aligned}
$$

Since $\Im\left(q_{c}\right)=b_{c}$ and $\Im\left(q_{m}\right)=b_{m} \omega^{-\gamma}$, we have easily the following estimate:

$$
b_{c} \omega^{2}\|w\|_{L^{2}(\mathcal{O})}^{2}+b_{m} \omega^{2-\gamma}\|w\|_{L^{2}\left(\mathcal{O}_{\delta}\right)}^{2} \leq C\left\|\mathrm{R}_{0} v\right\|_{L^{2}\left(\mathcal{O}_{\delta}\right)}\|w\|_{L^{2}\left(\mathcal{O}_{\delta}\right)} .
$$

Using Lemma 3.2 and estimate (26), we infer successively:

$$
\begin{aligned}
\|w\|_{L^{2}\left(\mathcal{O}_{\delta}\right)} & \leq C \omega^{3 \gamma-2} \sqrt{\delta}\left(\left.|v|_{\partial \mathcal{O}}\right|_{L^{2}(\partial \mathcal{O})}+|\mathfrak{g}|_{L^{2}(\mathbb{T})}\right) \\
& \leq C \omega^{3 \gamma-2} \sqrt{\delta}|\mathfrak{g}|_{H^{1 / 2}(\mathbb{T})}
\end{aligned}
$$

then

$$
\|w\|_{L^{2}(\mathcal{O})} \leq C \omega^{\frac{5 \gamma-4}{2}} \sqrt{\delta}|\mathfrak{g}|_{L^{2}(\mathbb{T})},
$$

which proves estimate (51). Suppose $\gamma \in(2 / 3,1]$ and that there exists a constant $c \in(0,1)$ such that for all $k \in \mathbb{Z}$,

$$
\left|k^{2} / \omega^{2}-a_{m}\right| \geq c \omega^{-1 / 2}
$$

According to (27),

$$
\left\|\mathrm{R}_{0} v\right\|_{L^{2}\left(\mathcal{O}_{\delta}\right)} \leq C \omega\|v\|_{L^{2}\left(\mathcal{O}_{\delta}\right)},
$$

hence

$$
\|w\|_{L^{2}\left(\mathcal{O}_{\delta}\right)} \leq C \omega^{\gamma-1} \sqrt{\delta}|\mathfrak{g}|_{H^{1 / 2}(\mathbb{T})}
$$

then

$$
\|w\|_{L^{2}(\mathcal{O})} \leq C \omega^{\gamma / 2-1} \sqrt{\delta}|\mathfrak{g}|_{L^{2}(\mathbb{T})},
$$

which ends the proof of the theorem. 


\section{Discussion OF EXTENSIONS AND FUtURE DIRECTIONS}

A natural expansion of this paper consists in generalizing our boundary condition to a domain of an arbitrary shape. The analysis necessary to the proof involves the theory of pseudodifferential operators. When the curvature of the domain is not constant the pseudocalculus is much more tedious since the derivatives of the curvature lead to no trivial difficulties. However it is possible to obtain a formal impedance boundary condition using our factorization method.

Denote by $\kappa$ the curvature of $\partial \mathcal{O}$. Then Helmholtz operator $\mathscr{L}$ defined by $(12)$ equals:

$$
\mathscr{L}=\frac{1}{\delta^{2}} \partial_{\eta}^{2}+\frac{1}{(1+\delta \kappa \eta)^{2}} \partial_{\theta}^{2}+q_{m} \omega^{2}+\frac{\kappa}{1+\delta \kappa \eta} \frac{1}{\delta} \partial_{\eta}-\frac{\delta \kappa^{\prime} \eta}{(1+\delta \kappa \eta)^{3}} \partial_{\theta} .
$$

Using our factorization method, the symbol $d_{1}$ depends on $\theta$ and it equals:

$$
\left\{\begin{array}{l}
d_{1}(\omega, \eta, k, \theta)=\sqrt{\frac{k^{2}}{(1+\delta \kappa(\theta) \eta)^{2}}-q_{m} \omega^{2}}, \\
\Re\left(d_{1}(\omega, \theta, \eta, k)\right) \leq 0 .
\end{array}\right.
$$

The symbol $R_{1}$ is

$$
\begin{aligned}
R_{1} & =\mathscr{L}-\left(\frac{1}{\delta} \partial_{\eta}+D_{1}\right)\left(\frac{1}{\delta} \partial_{\eta}-D_{1}\right), \\
& =\mathscr{L}-\left(\frac{1}{\delta^{2}} \partial_{\eta}^{2}-\frac{1}{\delta}\left[\partial_{\eta}, D_{1}\right]-D_{1}^{2}\right) .
\end{aligned}
$$

Note that the symbol of $D_{1}^{2}$ is not exactly $d_{1}^{2}$. Here is the first diffulcty, since we have to determine the leading term of $\sigma_{D_{1}^{2}}$. If $\gamma=0$, it is exactly $d_{1}^{2}$ but as soon as $\gamma>0$, it is not necessary true for all the frequency range we consider. However, we are confident that there exists a condition similar to (10) such that the symbol $r_{1}$ defined by $r_{1}=\sigma_{D_{1}^{2}}-d_{1}^{2}$ is of lower order than $\sigma_{D_{1}^{2}}$. Defining $s_{1}$ by

$$
s_{1}=\sigma_{R_{1}}-\frac{\mathrm{i} \tau \kappa}{\delta(1+\delta \kappa \eta)},
$$

we obtain the new symbols $d_{0}$ and $\widetilde{d}_{0}$ :

$$
\begin{aligned}
& \tilde{d}_{0}=-\frac{s_{1}}{2 d_{1}}+\frac{\kappa}{2(1+\delta \kappa \eta)}, \\
& d_{0}=-\frac{s_{1}}{2 d_{1}}-\frac{1}{2(1+\delta \kappa \eta)} .
\end{aligned}
$$

and we set

$$
d=d_{1}+d_{0}, \quad \tilde{d}=d_{1}+\widetilde{d}_{0} .
$$

Therefore the definitions (5) of $\mathscr{A}$ and $\mathscr{B}$ with the new symbols $d$ and $\widetilde{d}$ lead formally to a generalize impedance boundary condition similar to (8), which involves the curvature of $\partial \mathcal{O}$.

As we said above, the $L^{2}$-error estimates due to this boundary condition are difficult to obtain. Another difficulty comes from the numerical simulations. Actually, so that an finite element method might be used for the computations, it is necessary to approach the pseudodifferential boundary condition by differential operators. To obtain such approximation, we expect that appropriate expansions 
of the above symbols in power of $k$ have to be found, and the errors of this new approximation have to be proved.

\section{ACKNOWLEDGEMENTS}

The former author thanks very warmly O.Lafitte for his well-considered advice and suggestions.

\section{REFERENCES}

[1] Habib Ammari and Sailing He. Effective impedance boundary conditions for an inhomogeneous thin layer on a curved metallic surface. IEEE Transactions on Antennas and Propagation, 46(5):710-715, 1998.

[2] Elena Beretta, Elisa Francini, and Michael S. Vogelius. Asymptotic formulas for steady state voltage potentials in the presence of thin inhomogeneities. A rigorous error analysis. J. Math. Pures Appl. (9), 82(10):1277-1301, 2003.

[3] Elena Beretta, Arup Mukherjee, and Michael Vogelius. Asymptotic formulas for steady state voltage potentials in the presence of conductivity imperfections of small area. Z. Angew. Math. Phys., 52(4):543-572, 2001.

[4] L. A. Carvalho dos Santos and J. Hounie. Estimates for the Poisson kernel and Hardy spaces on compact manifolds. J. Math. Anal. Appl., 299(2):465-493, 2004.

[5] B. Engquist and J.C. Nédélec. Effective boundary condition for acoustic and electromagnetic scattering thin layer. Technical Report of CMAP, 278, 1993.

[6] Derek J. Hansen, Clair Poignard, and Michael S. Vogelius. Asymptotically precise norm estimates of scattering from a small circular inhomogeneity. Appl. Anal., 86(4):433-458, 2007.

[7] Olivier Lafitte and Gilles Lebeau. Équations de Maxwell et opérateur d'impédance sur le bord d'un obstacle convexe absorbant. C. R. Acad. Sci. Paris Sér. I Math., 316(11):1177-1182, 1993.

[8] Olivier D. Lafitte. Diffraction in the high frequency regime by a thin layer of dielectric material. I. The equivalent impedance boundary condition. SIAM J. Appl. Math., 59(3):1028-1052 (electronic), 1999.

[9] M. A. Leontovitch. Approximate boundary condition for the electromagnetic field on the surface of a good conductor. Investigations on Radiowave Propagation, PtII, Printing House of the Acad. of Science, pages 5-12, 1948.

[10] Clair Poignard. Asymptotics for steady state voltage potentials in a bidimensionnal highly contrasted medium with thin layer. Math. Meth. Appl. Sci., July 2007. DOI: 10.1002/mma.923.

Centre de Mathématiques Appliquées, umr CNRS 7641, Ecole Polytechnique X, 91128 Palaiseau Cedex, France. 